\title{
Passive prosthetic ankle-foot mechanism for automatic adaptation to sloped surfaces
}

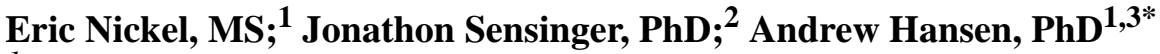 \\ ${ }^{1}$ Minneapolis Department of Veterans Affairs Health Care System, Minneapolis, MN; ${ }^{2}$ Prosthetic Design and Control \\ Laboratory, Rehabilitation Institute of Chicago, Chicago, IL, and Departments of Physical Medicine and Rehabilitation \\ and Mechanical Engineering, Northwestern University, Evanston, IL; ${ }^{3}$ Program in Rehabilitation Science, University of \\ Minnesota, Minneapolis, $M N$
}

\begin{abstract}
This article describes the development of a prototype prosthetic ankle-foot system that passively adapts to surface slopes on each step of walking. Engineering analyses were performed to design the cam clutch and clutch engagement and disengagement mechanism. The prototype was tested by a veteran with a unilateral transtibial amputation. Kinematic and kinetic data were recorded while the subject walked on a treadmill at slopes ranging from -10 to +10 degrees. After each slope condition, the subject rated his level of exertion and socket comfort. The subject reported increased comfort and reduced exertion for downhill slopes when using the prototype compared with his usual prosthesis. The subject also expressed that when walking downhill on the prototype, it was the most comfortable he had ever been in a prosthesis. The prosthetic ankle torque-angle relationship shifted toward dorsiflexion for uphill and toward plantar flexion for downhill slopes when using the prototype, indicating slope adaptation, but this effect did not occur when the subject walked with his usual prosthesis. The prototype also demonstrated late-stance plantar flexion, suggesting the potential for storing and returning more energy than standard lower-limb prostheses.
\end{abstract}

Key words: adaptation, ankle, equilibrium point, foot, gait, passive prosthetic, prosthesis, ramps, slope, walking.

\section{INTRODUCTION}

When nondisabled persons walk on sloped or uneven surfaces, they make adjustments to the kinematics and kinetics of their gait patterns [1-4]. According to Hansen et al., the net result of kinematic changes can be viewed as a change in ankle alignment to account for the change in surface slope [1]. Furthermore, in nondisabled persons, the first step on a different surface is adapted to that surface [5-6]. But without direct neural control, persons with lower-limb amputations do not have the ability to adjust their prosthesis for every step. Failure to adapt to terrain can lead to increased peak loading in the socket, discomfort, and tissue damage on the residual limb [7] and may contribute to falls when an unexpected new surface is encountered. To realize a truly adaptive prosthesis, the alignment of the ankle must be set independently for each step based only on the mechanics of that step and the surface encountered.

\footnotetext{
*Address all correspondence to Andrew H. Hansen, PhD; Minneapolis VA Health Care System, One Veterans Drive (Research-151), Minneapolis, MN 55417; 612-467-2910; fax: 612-725-2093. Email: andrew.hansen2@va.gov http://dx.doi.org/10.1682/JRRD.2013.08.0177
} 
There are some nonadaptive lower-limb prostheses that are tolerant of terrain variance through the use of multiaxial deflecting elements or ranges of hydraulic damping placed in series with high stiffness foot plates or keels (e.g., Epirus and Echelon feet from Endolite; Miamisburg, Ohio). The physical stops at the end of the range of motion of these features still engage the high stiffness property at the same ankle angle regardless of surface slope, so the ankle torque-angle relationship for the high stiffness element remains at a single equilibrium point.

Some microprocessor-controlled lower-limb prostheses are capable of changing alignment automatically, yet these systems often require incremental adjustments over several steps before adaptation is completed. Although these prostheses do adapt their alignment to surface slopes, they are not able to adjust for the first step on a new surface or for an uneven surface that is constantly changing slope.

Some historical prosthetic foot-ankle systems were developed that provide first-step adaptation; however, these devices were not clinically viable. For example, Hans Mauch developed a passive mechanical prosthetic ankle that adapted to sloped surfaces [8]. The mechanism relied on gravity to hold a ball at the nadir of a track such that when the shank reached vertical the ball closed a port, essentially "locking" the ankle. This approach was well received by the test users; however, rotary hydraulic systems are difficult to seal properly and the device was discontinued because of hydraulic fluid leakage and the need for frequent maintenance. Hansen et al. have also developed two prototype foot-ankle systems that adapt on each step of walking based on nondisabled walking characteristics [9]. Williams et al. used a weight-activated knurled cam clutch to engage a high stiffness rubber bumper at foot flat [10]. The proof-of-concept prototype successfully demonstrated the plausibility of the concept, yet many improvements were needed, including refinement of the cam clutch mechanism and clutch engagement mechanism. Nickel et al. used a releasable wrap spring clutch mechanism to engage a high-stiffness rubber bumper at foot flat [11]. The prototype showed evidence of changing the equilibrium point in response to walking on sloped surfaces, but the clutch did not support sufficient torque.

Our goal for the present work was to refine the design by Williams et al. and bring the concept closer to a clinically viable device. The refinements were aimed at (1) reducing engagement/disengagement noise by using a smooth cam surface instead of the knurled cam, (2) simplifying the engagement/disengagement mechanism by using a hinge instead of a telescoping slider, (3) redesigning the system to fit within a commercially available cosmetic foot shell, and (4) general design enhancements for structural strength, reduced weight, and reliable performance.

\section{METHODS}

\section{Prototype Design}

The ankle-foot prototype of the present work (Figure 1) is designed to provide a step-specific equilibrium point, or set-point, setting the dorsiflexion-plantarflexion alignment (zero-torque angle) on each step based on the surface slope encountered. The prototype is designed to achieve this feature through the refinement of three critical elements: (1) high-stiffness roll-over, (2) low-stiffness plantarflexion and neutralization, and (3) high-stiffness element engagement and disengagement.

The high-stiffness roll-over is achieved through the use of a deflecting foot plate and a specially designed rigid keel ring (Figure 2). The foot plate is a flat $10 \mathrm{~mm}$ thick sheet of nylon 6/6 (McMaster Carr; Chicago, Illinois) with

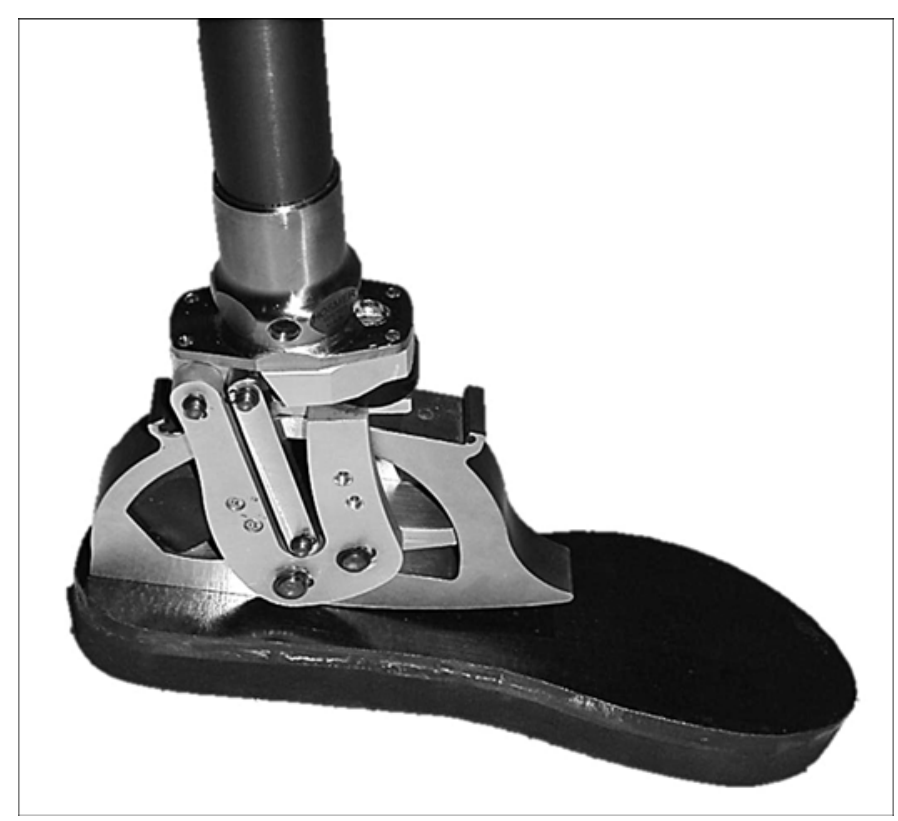

Figure 1.

Prototype of prosthetic ankle-foot system with step-specific equilibrium point. 


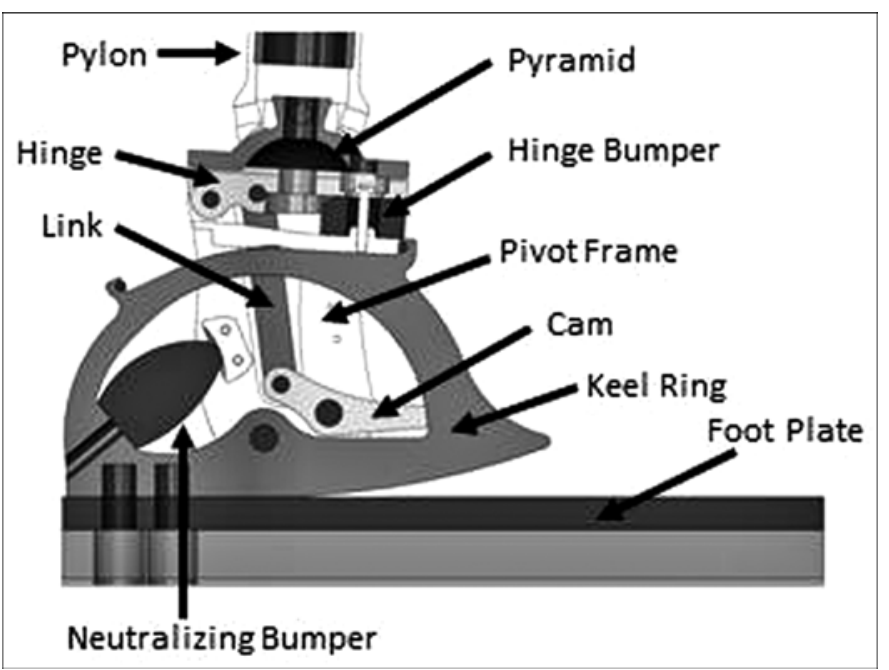

Figure 2.

Computer design cut-away view of prototype with main parts labeled.

a layer of black crepe for cushioning and a layer of rubber tread for traction. The keel ring is machined from maraging steel, a high-strength precipitation-hardened steel superalloy. The bottom arc of the keel ring is designed to form a natural ankle-foot rocker shape for level ground walking [12], taking into account the engagement of the flexible foot plate at foot flat. The keel design allows the foot plate to flex until it conforms to the keel rocker shape but no further, even if the user were to carry additional weight, better matching the function of the nondisabled ankle-foot system [13].

The low stiffness plantarflexion and neutralization are achieved through the use of a polyurethane rubber bumper (neutralizing bumper in Figure 2) placed within the keel ring. The neutralizing bumper is seated within the posterior portion of the ring interior. The bumper is placed between the keel ring and the posterior portion of the pivot frame such that as the foot plate and keel ring are rotated in the direction of plantarflexion, the neutralizing bumper is compressed. The durometer of the neutralizing bumper is 25 Shore A and provides an effective ankle stiffness of $0.31 \mathrm{~N} \cdot \mathrm{m} /{ }^{\circ}$. Preliminary mechanical testing indicates this stiffness is sufficient to reduce plantarflexion velocity (reducing foot slap).

The high stiffness engagement and disengagement is achieved through the use of a weight-activated cam clutch. The hinge is designed to rotate under load such that when the user walks on the prototype, the load they apply through the prosthesis compresses the hinge bumper (60 Shore A polyurethane rubber). This rotation of the hinge relative to the pivot frame results in downward translation of the links, which in turn rotates the cam into contact with the keel ring. After foot flat, when the cam is in contact with the keel ring, the pivot frame is unable to rotate in the direction of dorsiflexion but is free to rotate in the direction of plantarflexion. With the pivot frame unable to rotate in the direction of dorsiflexion, the loads are transferred through the structure to the footplate, which deflects, storing and returning energy from foot flat to toe off. Upon unloading, the hinge bumper forces the hinge to open, pulling the cam away from the keel ring.

During a full cycle of walking with the prototype, several distinct events occur. First, during early stance, the neutralizing bumper compresses as the pivot frame rotates from a neutral position ( $3^{\circ}$ of dorsiflexion) into plantarflexion (Figure 3(a)-(b)) until the foot plate comes into full contact with the surface (i.e., foot flat shown as $15^{\circ}$ of plantarflexion in Figure 3(b)). As the user applies load to the prosthesis, the hinge is compressed, rotating the cam into contact with the keel ring. Once the cam is in contact with the keel ring and the user begins to roll over the ankle (moving the shank in the direction of dorsiflexion), the clutch is engaged and the pivot frame orientation is locked onto the keel ring (at the $15^{\circ}$ of plantarflexion in Figure 3(b)). The angle of the pivot frame relative to the keel ring at this time is considered to be the set point or equilibrium point and should change with the surface orientation for each particular step. The clutch is locked as the user rolls over the foot (Figure 3(b)-(d)), with the foot plate deflecting up to the bottom of the keel ring (Figure 3(c)-(d)). As the user unloads the prosthesis (Figure 3(d)-(e)), the foot plate returns to its step-specific equilibrium point, which includes the plantarflexion present at the time the clutch locked ( $15^{\circ}$ of plantarflexion in Figure 3 ), restoring energy to the user. When fully unloaded, the hinge bumper forces the hinge open, disengaging the clutch and allowing the neutralizing bumper to return the prosthesis to a neutral ( $3^{\circ}$ of dorsiflexion) alignment for swing phase (Figure 3(e)-(f)).

Two aspects of the design were critical for achieving slope adaptation. First, the cam clutch needed to jam under realistic surface conditions. Second, the engagement/disengagement mechanism needed to reliably engage when loaded by the user's body weight yet also reliably disengage when unloaded. 
(a)

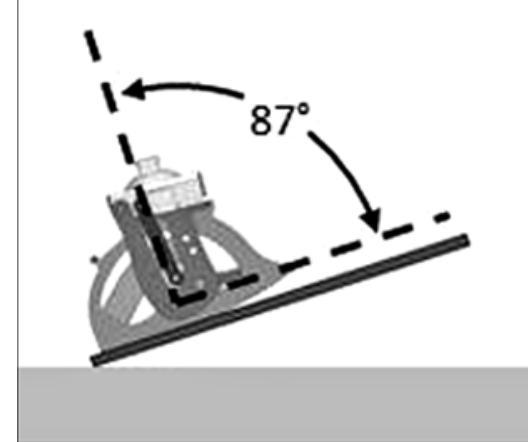

(d)

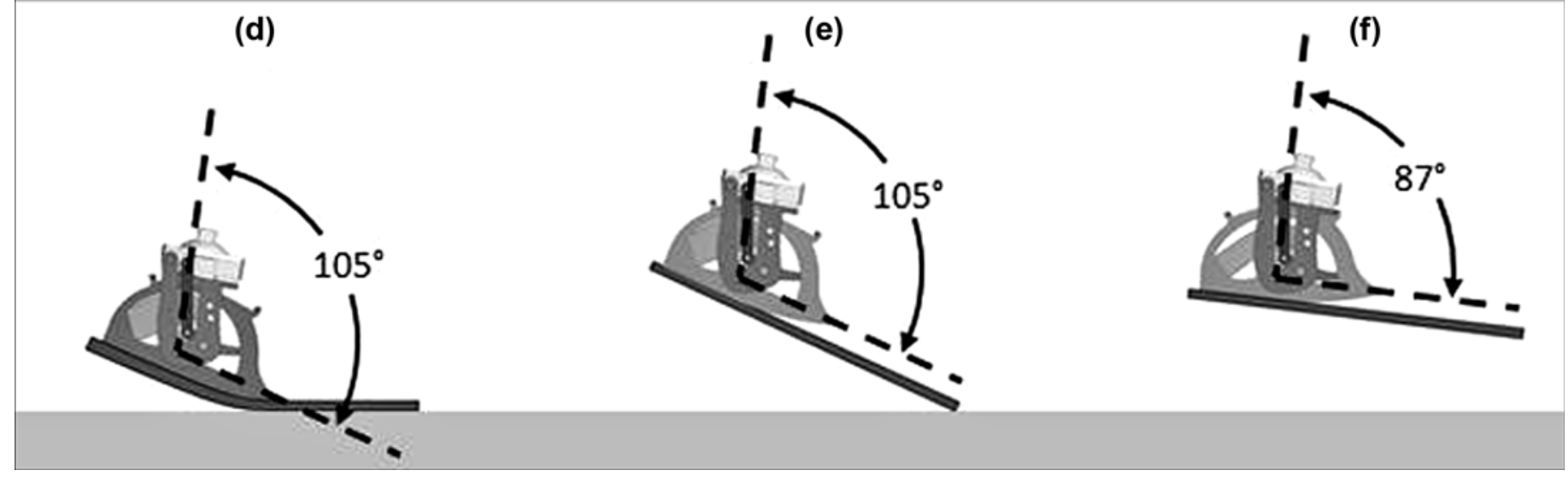

(b)

(c)

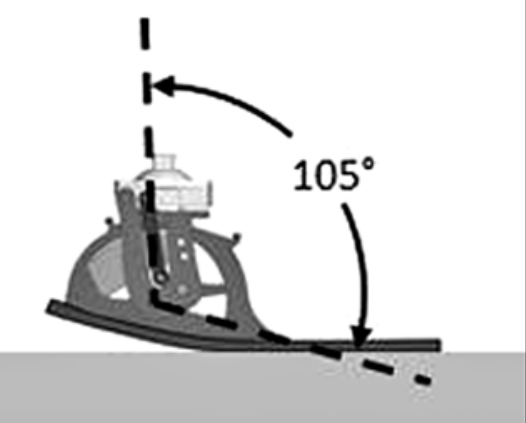

(f)

Figure 3.

Functional progression of prototype through one step. Dashed lines indicate orientation of pylon and keel ring, providing visual depiction of clutch angle. (a) Foot is in neutral position as heel makes contact with surface. (b) Foot plate and keel ring plantarflex until foot plate has conformed to walking surface. As user loads prosthetic limb and begins to roll over foot, cam clutch engages and roll-over stores energy in deflection of foot plate (c and $\mathbf{d}$ ), then returns that energy to user during unloading (d to $\mathbf{e}$ ). When prosthetic limb is unloaded, cam is disengaged, allowing neutralizing bumper to return foot plate and keel ring to neutral or dorsiflexed position for swing phase.

For a cam clutch to grip effectively, the tangent of the jam angle, or angle of contact between the cam and keel ring, must be less than the lowest coefficient of static friction between the two surfaces during use (Equations 1-6 and Figure A1 in the Appendix, available online only). This ensures sufficient normal force at the interface between the cam and the keel ring that friction is able to arrest motion of the cam in the direction of dorsiflexion. The cam is designed to have an area of contact with the keel ring to better distribute the contact stresses. This contact area spans a range of jam angles from $7^{\circ}$ to $12^{\circ}$. At the worst, with the load primarily at the $12^{\circ}$ end of the contact face, the clutch would be able to remain jammed at a coefficient of static friction as low as 0.21 . 
the cam to remain engaged under the internal torque produced by the neutralizing bumper. Under maximum plantarflexion and no applied body weight load, the hinge bumper force is transmitted through the links to the posterior aspect of the cam, countering some of the normal force at the interface between the cam and keel ring. The force that the hinge bumper must exert at the point of clutch disengagement can be determined by Equations 11-15 and Figure A3 in the Appendix.

These three competing factors must be balanced to provide a range of conditions under which the device can reliably operate. Figure 4 illustrates the relationship between the actual static coefficient of friction between the cam and keel ring surfaces and the range of hinge bumper forces that would result in engagement and/or disengagement. For low ankle stiffness in early stance (i.e., soft neutralizing bumpers, Figure 4(a)) the clutch will both engage and disengage over a range of hinge bumper forces and coefficients of friction; however, greater ankle stiffnesses, such as mean values based on nondisabled data from Palmer [16] (Figure 4(b) for moderate/fast walking), significantly reduce the viable range of hinge bumper forces and require tight control of the coefficient of friction to ensure disengagement.

The prototype developed in this work and used for testing had a mass of $1.49 \mathrm{~kg}$ and a build height (floor to top of
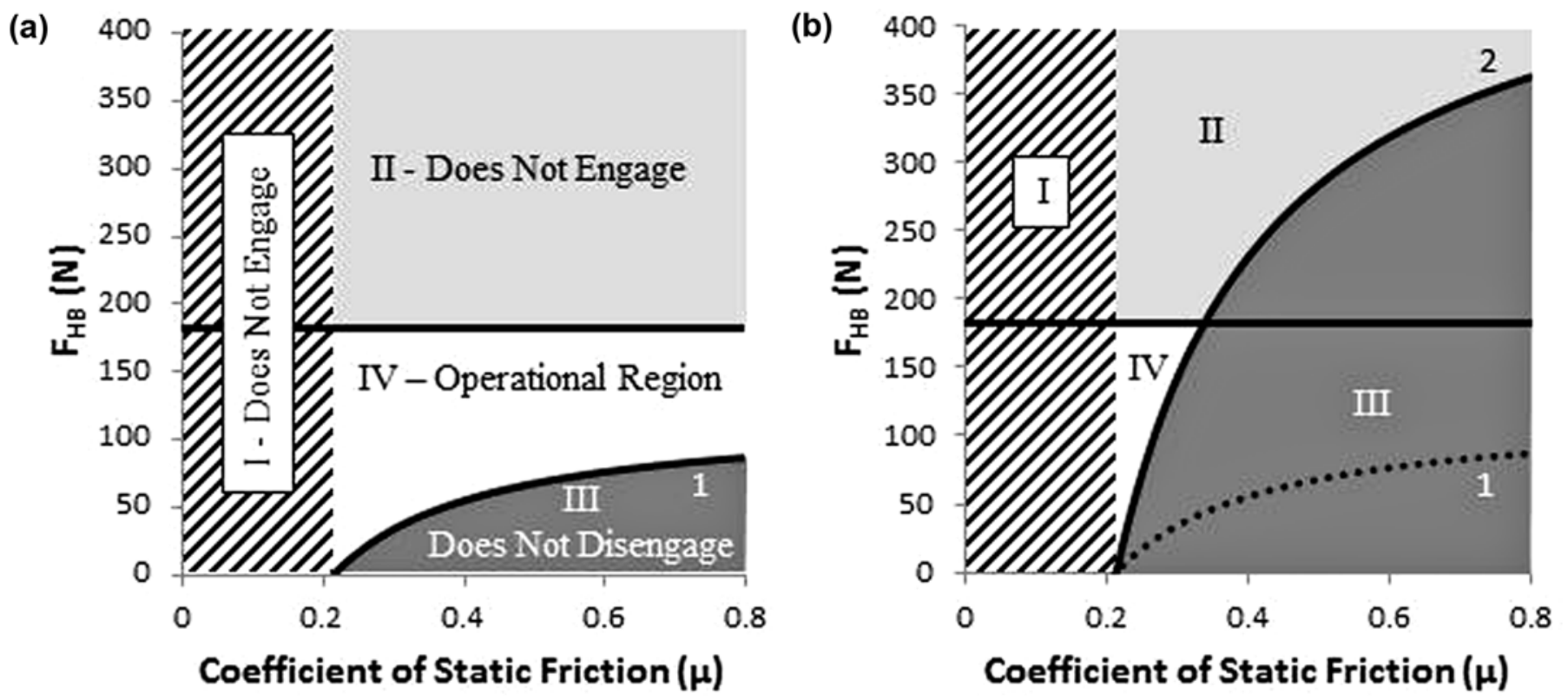

Figure 4.

Optimization diagram for prototype's engagement/disengagement mechanism. Force applied by hinge bumper at point of cam clutch engagement $\left(\mathrm{F}_{\mathrm{HB}}\right)$, coefficient of static friction between cam and keel ring $(\mu)$, and durometer of neutralizing bumper are critical parameters to successful engagement and disengagement of clutch. In region I, cam is not capable of engaging because coefficient of static friction is too low for clutch engagement at jam angle of $12^{\circ}$. Changing cam jam angle can change terminus of this region (which ends at tangent of cam jam angle). In region II, hinge bumper force $\left(\mathrm{F}_{\mathrm{HB}}\right)$ is too large relative to user's body weight to reliably engage clutch during standing and walking. Alterations to geometry of hinge can affect this region. In region III, hinge bumper is unable to overcome internal moment produced by neutralizing bumper at maximum plantarflexion and therefore is unable to force cam to disengage reliably. Altering durometer of neutralizing bumper can change area of this region, as shown in (b). Curve 1 indicates size of region III for neutralizing bumper used in testing and assuming $100 \mathrm{~kg}$ user and corresponds to region III of (a). Curve 2 in (b) indicates size of region III for neutralizing bumper that produces ankle stiffness of $0.015 \mathrm{~N} \cdot \mathrm{m} / \mathrm{kg} \cdot{ }^{\circ}$, based on mean data from Palmer [16] for nondisabled persons walking at moderate or fast walking speeds. Region IV is operational region of parameter space in which clutch is able to both engage and disengage reliably during standing and walking. Curves 1 and 2 shown in (b) demonstrate reduced sensitivity to neutralizing bumper durometer (and therefore ankle rotational stiffness) when coefficient of static friction approaches boundary of region 1. 
pyramid adaptor) of $15.4 \mathrm{~cm}$. The nylon foot plate was bonded to a layer of crepe and rubber tread, so a cosmetic cover and shoe were not needed when testing the prototype.

\section{Human Subject Testing}

The prototype was pilot tested on one veteran with unilateral below-knee amputation. He was 29 yr old with a mass of $85.8 \mathrm{~kg}$ and had 8 mo of experience walking with a prosthesis, including trials of several feet ranging from passive carbon fiber springs to power-generating, microprocessor-driven devices. The subject was an active walker and runner. His usual prosthesis was an Ottobock 1C62 Triton foot with a Harmony elevated vacuum suspension system (Duderstadt, Germany). Data were collected while the subject walked at a comfortable selfselected speed $(1.0 \mathrm{~m} / \mathrm{s})$ on a split-belt instrumented treadmill (Bertec Corporation; Columbus, Ohio). The subject's movements were tracked using an 8-camera motion analysis system (Qualisys AB; Gothenburg, Sweden) and reflective markers placed over anatomical landmarks on the lower limbs. The subject walked on the treadmill for $30 \mathrm{~s}$ at each of five different slope conditions: level and both uphill and downhill at angles of $5^{\circ}$ and $10^{\circ}$ using first the prototype, followed by all conditions repeated using his usual foot. The walking speed was the same for all conditions. After walking on the treadmill at each slope condition, the subject was asked to rate his socket comfort and walking effort using the Socket Comfort Score and Rating of Perceived Exertion, respectively. The Socket Comfort Score is an 11-point scale, where a score of 10 indicates the greatest imaginable comfort and a score of 0 indicates the worst comfort imaginable [17]. The Rating of Perceived Exertion is a numeric scale that asks subjects to rate the level of effort they feel they are exerting, where a rating of 0 indicates absolutely no exertion and a rating of 10 indicates the maximum possible exertion [18].

\section{Data Analysis}

Our motion analysis system tracked reflective markers placed on the subject and output the coordinates of each marker in the global laboratory frame of reference. These coordinates were then imported into MATLAB (MathWorks; Natick, Massachusetts), where custom scripts were created to calculate joint centers, joint torques, and joint angles. Joint torque at the ankle was estimated using a quasistatic approach (force times its perpendicular distance from the ankle joint), a technique that provides nearly identical results to an inverse dynamics approach for the ankle [19]. Clinically significant differences in socket comfort score are two points or more [17]. Clinically significant differences in perceived exertion are any difference of two or more categories [18]. Categories occur at 0 , $0.5,1,2,3,4,5,7,9$, and 10 .

\section{RESULTS}

We included from 16 to 26 strides in the analysis for different experimental conditions. The strides for each experimental condition were averaged together. These results are shown in Figure 5.

When using the prototype, the ankle angle followed a similar trajectory during stance phase for all slopes, though it has been shifted by the magnitude and direction of the slope (Figure 5(a)). Furthermore, during late stance $(60 \%-70 \%$ of the gait cycle), there was plantarflexion of the ankle as the foot plate was unloaded. This plantarflexion resulted from the initial ankle plantarflexion during early stance and approached the magnitude of the early stance plantarflexion. When the subject was using his usual prosthesis (Figure 5(b)), the ankle angle trajectory varied widely between slope conditions and there was no late stance plantarflexion.

The ankle torque-angle plots (Figure 5(c)-(d)) are a concise way to identify the equilibrium point of the prosthesis and any changes to the equilibrium point. When using the prototype, the subject's ankle torque-angle curves were separated along the ankle angle axis. When the subject was using his usual prosthesis, the ankle torque-angle curves occurred along the same trajectory with different slope conditions utilizing different regions of the trajectory.

When using the prototype, the subject reported greater socket comfort than when using his usual prosthesis on four out of five slope conditions (Figure 6(a)). On an incline of $5^{\circ}$, he rated his socket comfort the same for both feet.

The subject reported that he used less effort when walking on declined surfaces when using the prototype than when using his usual prosthesis (Figure 6(b)). The difference in perceived exertion on declines was clinically significant. The effort used to walk on level and inclined surfaces were the same for both feet.

The subject also had several comments about the prototype. The subject felt that the neutralizing bumper used for testing was too soft and a stiffer bumper may improve 
(a)

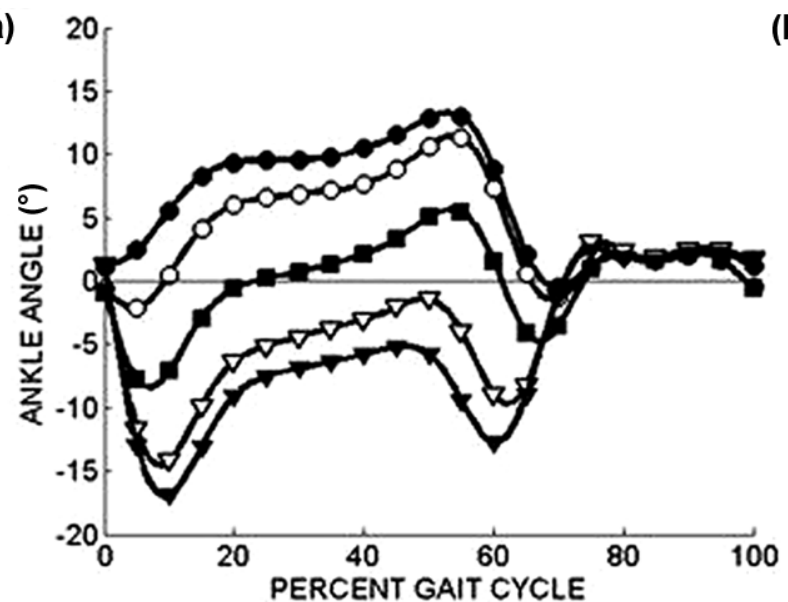

(c)

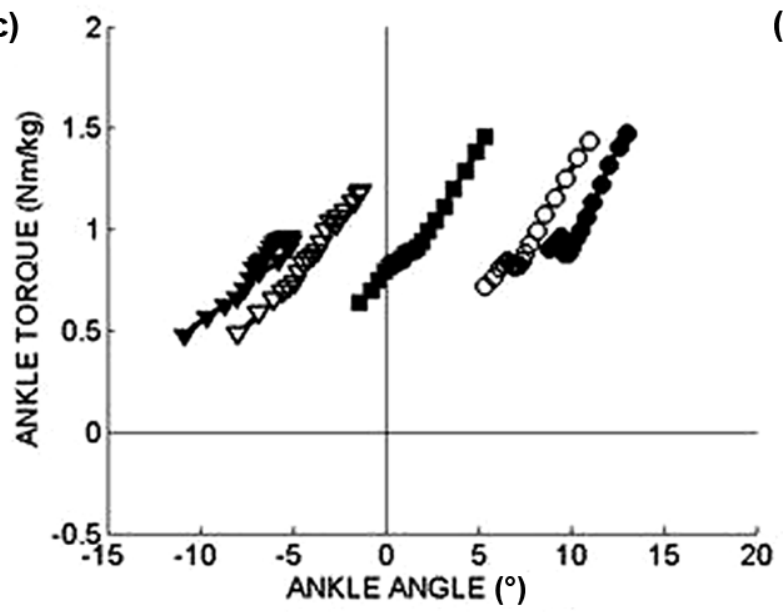

(b)

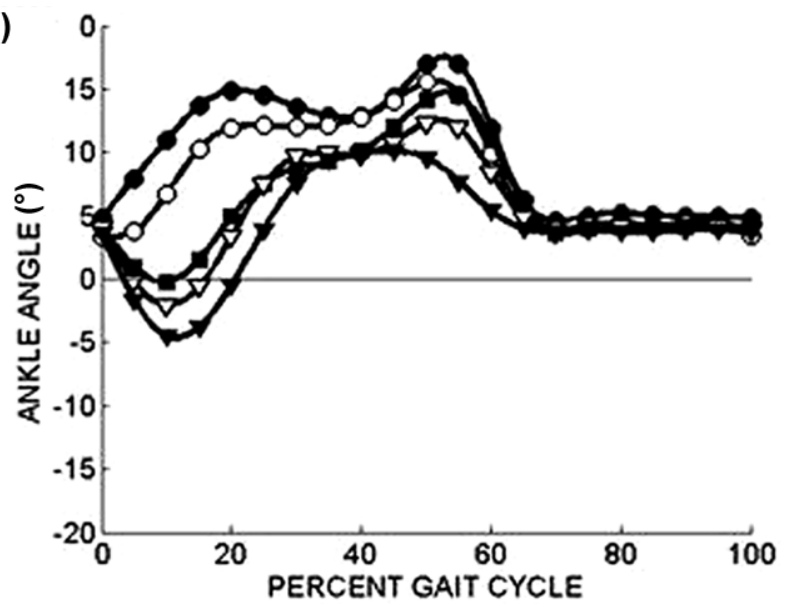

(d)

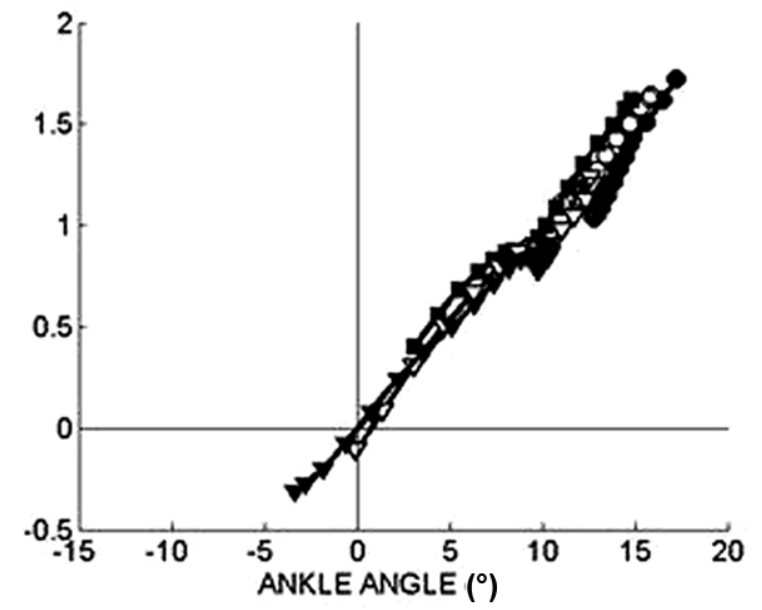

\section{-Up10 deg - - Up5 deg $\rightarrow$ - Level $\rightarrow$-Down5 deg $\rightarrow$-Down10 deg}

Figure 5.

Ankle kinematic and kinetic data for prototype and subject's usual prosthesis. Prototype ((a) and (c)) demonstrates several expected indicators of slope adaptation, including consistent ankle angle trajectory shape that is shifted into dorsiflexion or plantarflexion during stance phase for different slopes and ankle torque-angle curves that are separated along ankle angle axis indicating different equilibrium points for each slope condition. Subject's usual prosthesis ((b) and (d)) demonstrates varied ankle angle trajectories when walking on different slopes and ankle torque-angle relationships during single-limb support that overlay one another, indicating single equilibrium point.

comfort in early stance. He felt that the prototype was best when at a particular stride length, with steps that were too short or too long encountering resistance. The prototype was very comfortable when standing on sloped surfaces, and when walking downhill it was the most comfortable he had ever been on a prosthetic foot. In general, the subject felt less pressure in the socket when using the prototype and did not need to use his knee to fight socket pressures when walking on slopes. The subject liked the instant response to changes in surface slope and appreciated the stability produced by having the footplate in full contact with the ground when standing, especially on slopes.

\section{DISCUSSION}

When using the prototype prosthesis, there were several indicators of successful slope adaptation. For example, the ankle angle trajectory maintained essentially the 
(a) Socket Comfort Score

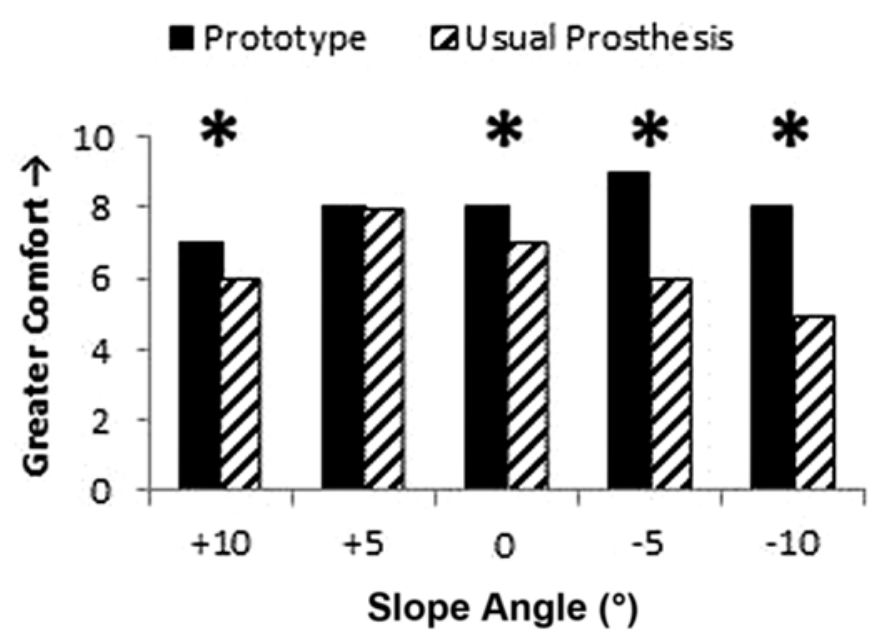

(b) Rating of Perceived Exertion

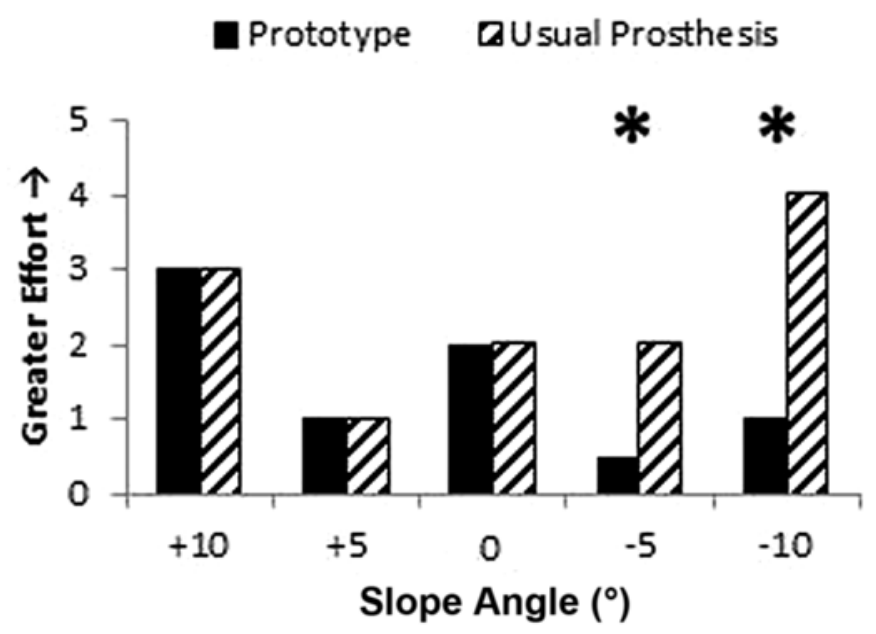

Figure 6.

Socket comfort and perceived exertion when using prototype and usual prosthesis. Subject reported clinically significant improvements (denoted by asterisks) in both comfort and exertion when walking on declines.

same shape but was shifted to follow the surface slope. The constant ankle angle during swing phase for all slope conditions further indicated that the prototype returns to a consistent neutral position after each step, suggesting that it makes the alignment adaptation independently on each and every step of walking. This adaptation can also be seen in the ankle torque-angle plot (Figure 5(c)) where the different slope conditions are a series of curves with similar magnitude and shape spread out along the ankle angle axis. These characteristics are in contrast to the subject's usual prosthesis that, although highly dynamic, shows widely dissimilar ankle angle trajectories when walking on surfaces of different slopes as well as ankle torque-angle relationships that overlay one another. This behavior is characteristic of a device that only has a single set-point and has a direct relationship between ankle angle and ankle torque.

Furthermore, the prototype prosthesis was able to provide late-stance plantarflexion, which the subject's usual prosthesis was unable to do. Current passive mechanical prostheses could more closely mimic the stance phase function of the nondisabled ankle-foot system during walking if they were more flexible and aligned in plantarflexion. However, a plantarflexed alignment is unsafe for the swing phase of walking. The prototype allows for two alignments set independently for stance phase and swing phase, with the stance phase alignment set at foot flat. With the prototype ankle-foot system, the energy stored in the foot plate is stored from a plantarflexed position and when unloaded is returned back to that plantarflexed position, providing a larger deflection and therefore potentially storing and returning more energy to the user than a traditional foot plate. After toe-off, the energy stored in the neutralizing bumper during early stance is used to return the keel ring and foot plate to a neutral or dorsiflexed position for toe clearance during swing phase. The enhanced energy storage and return properties of the prototype ankle-foot system may reduce the energy cost during walking for its users and should be investigated in future work.

The subject also experienced greater socket comfort and reduced effort when walking on declines, with no clinically significant difference on level and inclined conditions. This result matched well with his verbal comments pertaining to his sense of comfort when walking on declines. Further comments indicated that there were several additional features of the device that were appreciated by the subject. He liked the stability of the ankle when standing because the foot was completely on the ground regardless of the surface angle. This effect may also translate to seated positions where the ankle is free to plantarflex to accommodate the surface under low loads, though this was not mentioned by the subject. The subject also stated that when he falls it is mostly because 
of unexpected changes in the terrain, such as unexpectedly stepping onto a new surface elevation or stepping on a small object lying on the floor. A prosthesis that can adapt to unexpected surface orientations on every step of walking may be able to reduce the occurrence of falls in persons with lower-limb amputations.

Use of a smooth cam surface appeared to reduce but not eliminate engagement/disengagement noise. The source of the remaining noise was not readily identifiable. Furthermore, the amount of noise reduction could not be measured because the original prototype was no longer available for comparison. Further noise reduction efforts may be necessary to enhance the user experience for commercialization.

The engagement/disengagement mechanism for the prototype was simpler than the previous mechanism from Williams et al. [10]. There were fewer parts and a lower profile combined with a more rugged structure for improved durability.

The overall system was designed to fit within a Venture cosmetic foot shell (College Park Inc; Warren, Michigan). The parts all fit within the foot shell, though the prototype had a greater build height than the Venture foot and therefore extended out through the top of the foot shell. During maximal plantarflexion, the pivot frame did infringe on the cosmetic envelope in the posterior region of the shell. Redesigning the system to change the ankle pivot point (presently located in the same position as the Venture axis, just above the foot plate) to a higher position on the ankle would eliminate the infringement on the cosmetic envelope.

The structures were designed to be more rigid and durable than in previous versions, with all stress analyses being based on the fatigue strength of each part and displacement analyses being used to evaluate the effect of deflection under load prior to accepting part designs. The mass of the prototype was not optimized; thus, there was essentially no weight saving over previous prototypes, though new parts were designed to evaluate the mass of a next-generation weight-saving version and the resulting system could have a mass that is reduced from the present $1.49 \mathrm{~kg}$ (as tested, without cosmetic cover or shoe) to $1.04 \mathrm{~kg}$ without degrading performance or durability, and the potential for even further mass savings with more extensive redesign.

There are several limitations to this work. The parts were designed to be relatively easy to fabricate in small quantities; therefore, the shape and materials were not optimized. Also, the foot plate is a simple plastic plate and is not designed to be an efficient energy storage element. Further development and refinement to reduce weight, develop a better foot plate, and make the engagement/disengagement mechanism more robust are needed. The cam showed contact wear only on the surface region with the highest jam angle, indicating the effective jam angle for the clutch was essentially $12^{\circ}$. Deflection of the structures could be altering the distance between the cam pivot and the keel ring surface, concentrating the contact load at the $12^{\circ}$ jam angle end of the cam face. Under the limited use during the course of this study, the clutch held reliably, but in the future it may be necessary to redesign the cam with a maximum jam angle of $11^{\circ}$ or $10^{\circ}$ if the coefficient of friction lowers as the clutch wears during long-term testing. The prototype was also tested in only one subject. Although we feel this subject was an ideal candidate for pilot testing the prototype, future testing should be conducted with more subjects.

Additionally, the high stiffness engagement/disengagement mechanism is sensitive to the stiffness of the neutralizing bumper. The Shore A durometer of the neutralizing bumper is a critical aspect of the system and can have profound effects on several key features, including the perceived support during loading, standing balance, and clutch disengagement. The mass-normalized earlystance ankle stiffness for the subject was $0.0036 \mathrm{~N} \cdot \mathrm{m} / \mathrm{kg} \cdot{ }^{\circ}$ using the 25 Shore A durometer neutralizing bumper. We compared this value with the mass-normalized ankle torque for nondisabled persons based on data from Palmer [16] for individuals walking slow, normal, and fast (normalized mean 0.012, 0.015, and $0.015 \mathrm{~N} \cdot \mathrm{m} / \mathrm{kg} \cdot{ }^{\circ}$, respectively). The stiffness of the neutralizing bumper was well below the nondisabled means for our subject. Based on the subject's feedback, he felt the "heel" was soft but it was okay for him and there was no apparent "foot slap" problem when he was walking. A higher durometer neutralizing bumper would make for a "stiffer" heel but (as evidenced by Figure 4) would also reduce the range of hinge bumper stiffness and coefficient of friction between the cam and keel ring for which the engagement/ disengagement mechanism would function reliably. If tight control of the coefficient of friction over the life of the device (such as through lubrication or surface coatings) can be maintained, then the jam angle of the cam can be designed such that the clutch is barely able to engage, rendering the mechanism robust to the stiffness of the bumpers, allowing a sensitive hinge (soft hinge 
bumper) to be combined with a wide range of neutralizing bumpers.

\section{CONCLUSIONS}

The prototype prosthetic ankle-foot system demonstrated strong evidence of providing adaptation to surface slopes on every step of walking while improving comfort and reducing perceived exertion when walking downhill. It also provides late stance plantarflexion, which has the potential to provide enhanced energy storage and return. This prototype has demonstrated great potential, and further work should focus on refining the design, testing the durability of the system, and testing the function of the system in a larger number of persons with amputations.

\section{ACKNOWLEDGMENTS}

\section{Author Contributions:}

Mechanical design, modeling, and analysis: E. Nickel, J. Sensinger,

A. Hansen.

Study concept and design: A. Hansen.

Analysis and interpretation of data: A. Hansen.

Drafting of manuscript: E. Nickel, J. Sensinger, A. Hansen.

Institutional review coordination: E. Nickel.

Obtained funding: A. Hansen.

Financial Disclosures: The authors have declared that no competing interests exist.

Funding/Support: This material was based on work supported by the Department of Veterans Affairs, Veterans Health Administration, Rehabilitation Research and Development Service (pilot award \#A7656-P).

Additional Contributions: The authors would like to thank Sara Koehler, $\mathrm{PhD}$, for assistance with data collection and analysis; Steve Morin for his efforts in manufacturing and modifying parts for this project; Kyle Barrons, MSOP, for assistance fitting and aligning the prototype; and College Park Industries for providing a cosmetic foot covering for the project. Dr. Sensinger is now with the Institute of Biomedical Engineering and Electrical and Computer Engineering Department, University of New Brunswick, Fredericton, New Brunswick, Canada.

Institutional Review: The subject provided informed consent and the study was approved by the Minneapolis Department of Veterans Affairs Health Care System Institutional Review Board.

Participant Follow-Up: The authors do not plan to inform the participant of the publication of this article.

\section{REFERENCES}

1. Hansen AH, Childress DS, Miff SC. Roll-over characteristics of human walking on inclined surfaces. Hum Mov Sci. 2004;23(6):807-21. [PMID:15664674] http://dx.doi.org/10.1016/j.humov.2004.08.023

2. Leroux A, Fung J, Barbeau H. Postural adaptation to walking on inclined surfaces: I. Normal strategies. Gait Posture. 2002;15(1):64-74. [PMID:11809582] http://dx.doi.org/10.1016/S0966-6362(01)00181-3

3. Lay AN, Hass CJ, Gregor RJ. The effects of sloped surfaces on locomotion: A kinematic and kinetic analysis. J Biomech. 2006;39(9):1621-28. [PMID:15990102] http://dx.doi.org/10.1016/j.jbiomech.2005.05.005

4. Vrieling AH, van Keeken HG, Schoppen T, Otten E, Halbertsma JP, Hof AL, Postema K. Uphill and downhill walking in unilateral lower limb amputees. Gait Posture. 2008;28(2):235-42. [PMID:18242995] http://dx.doi.org/10.1016/j.gaitpost.2007.12.006

5. Prentice SD, Hasler EN, Groves JJ, Frank JS. Locomotor adaptations for changes in the slope of the walking surface. Gait Posture. 2004;20(3):255-65. [PMID:15531172] http://dx.doi.org/10.1016/j.gaitpost.2003.09.006

6. Ferris DP, Liang K, Farley CT. Runners adjust leg stiffness for their first step on a new running surface. J Biomech. 1999;32(8):787-94. [PMID:10433420] http://dx.doi.org/10.1016/S0021-9290(99)00078-0

7. Portnoy S, van Haare J, Geers RP, Kristal A, Siev-Ner I, Seelen HA, Oomens CW, Gefen A. Real-time subjectspecific analyses of dynamic internal tissue loads in the residual limb of transtibial amputees. Med Eng Phys. 2010; 32(4):312-23. [PMID:20074994] http://dx.doi.org/10.1016/j.medengphy.2009.12.006

8. Sowell TT. A preliminary clinical evaluation of the Mauch hydraulic foot-ankle system. Prosthet Orthot Int. 1981; 5(2):87-91. [PMID:7301527]

9. Hansen AH, Childress DS, Miff SC, Gard SA, Mesplay KP. The human ankle during walking: Implications for design of biomimetic ankle prostheses. J Biomech. 2004;37(10): 1467-74. [PMID:15336920] http://dx.doi.org/10.1016/j.jbiomech.2004.01.017

10. Williams RJ, Hansen AH, Gard SA. Prosthetic ankle-foot mechanism capable of automatic adaptation to the walking surface. J Biomech Eng. 2009;131(3):035002. [PMID:19154079] http://dx.doi.org/10.1115/1.3005335

11. Nickel EA, Hansen AH, Gard SA. Prosthetic ankle-foot system that adapts to sloped surfaces. J Med Dev. 2012; 6(1):011006. http://dx.doi.org/10.1115/1.4005787

12. Hansen AH. Roll-over characteristics of human walking with applications for artificial limbs [dissertation]. [Evanston (IL)]: Northwestern University; 2002. 
13. Hansen AH, Childress DS. Effects of adding weight to the torso on roll-over characteristics of walking. J Rehabil Res Dev. 2005;42(3):381-90. [PMID:16187250] http://dx.doi.org/10.1682/JRRD.2004.04.0048

14. Nadollek H, Brauer S, Isles R. Outcomes after trans-tibial amputation: The relationship between quiet stance ability, strength of hip abductor muscles and gait. Physiother Res Int. 2002;7(4):203-14. [PMID:12528576] http://dx.doi.org/10.1002/pri.260

15. Hlavackova P, Franco C, Diot B, Vuillerme N. Contribution of each leg to the control of unperturbed bipedal stance in lower limb amputees: New insights using entropy. PLoS ONE. 2011;6(5):e19661. [PMID:21603630] http://dx.doi.org/10.1371/journal.pone.0019661

16. Palmer ML. Sagittal plane characterization of normal human ankle function across a range of walking gait speeds [dissertation]. [Cambridge (MA)]: Massachusetts Institute of Technology; 2002.

17. Kendrick DB, Strout TD. The minimum clinically significant difference in patient-assigned numeric scores for pain. Am J Emerg Med. 2005;23(7):828-32. [PMID:16291435] http://dx.doi.org/10.1016/j.ajem.2005.07.009

18. Ries AL. Minimally clinically important difference for the UCSD Shortness of Breath Questionnaire, Borg Scale, and
Visual Analog Scale. COPD. 2005;2(1):105-10. [PMID:17136970]

http://dx.doi.org/10.1081/COPD-200050655

19. Wells RP. The projection of the ground reaction force as a predictor of internal joint moments. Bull Prosthet Res. 1981;10(35):15-19. [PMID:7332827]

Submitted for publication August 7, 2013. Accepted in revised form January 22, 2014.

This article and any supplementary material should be cited as follows:

Nickel E, Sensinger J, Hansen A. Passive prosthetic ankle-foot mechanism for automatic adaptation to sloped surfaces. J Rehabil Res Dev. 2014;51(5):803-14. http://dx.doi.org/10.1682/JRRD.2013.08.0177

ResearcherID/ORCID: Jonathon Sensinger, PhD: C-50122014

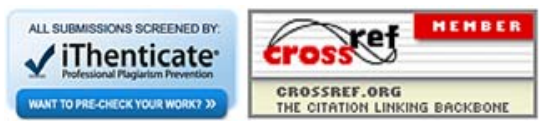


